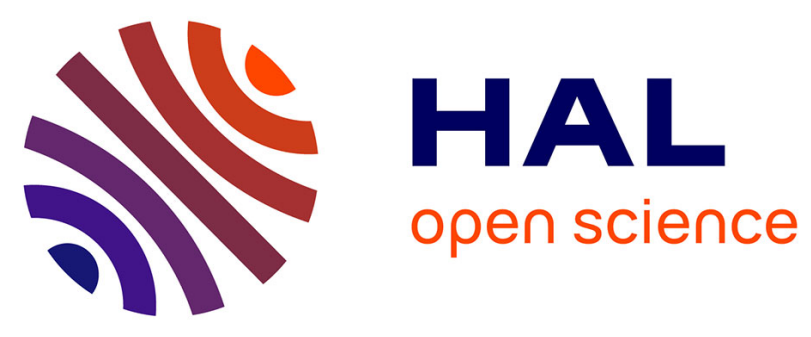

\title{
Wireless Measurement of the Pressure from the Ka-Band Radar Echo of a 3D-Printed Microfluidic Depolarizing Sensor
}

\author{
Timothee Marchal, Dominique Henry, Patrick Pons, Hervé Aubert
}

\section{- To cite this version:}

Timothee Marchal, Dominique Henry, Patrick Pons, Hervé Aubert. Wireless Measurement of the Pressure from the Ka-Band Radar Echo of a 3D-Printed Microfluidic Depolarizing Sensor. International Microwave Symposium (IMS 2021), Jun 2021, Atlanta, United States. 10.1109/IMS19712.2021.9574855 . hal-03279854

\section{HAL Id: hal-03279854 \\ https://hal.laas.fr/hal-03279854}

Submitted on 25 Oct 2021

HAL is a multi-disciplinary open access archive for the deposit and dissemination of scientific research documents, whether they are published or not. The documents may come from teaching and research institutions in France or abroad, or from public or private research centers.
L'archive ouverte pluridisciplinaire HAL, est destinée au dépôt et à la diffusion de documents scientifiques de niveau recherche, publiés ou non, émanant des établissements d'enseignement et de recherche français ou étrangers, des laboratoires publics ou privés. 


\title{
Wireless Measurement of the Pressure from the Ka-Band Radar Echo of a 3D-Printed Microfluidic Depolarizing Sensor
}

\author{
T. Marchal, D. Henry, P. Pons, H. Aubert \\ LAAS-CNRS, University of Toulouse
}

\begin{abstract}
In this paper, we report the remote measurement of the pressure from the Radar interrogation of a novel microfluidic passive sensor. This sensor includes a microfluidic band-pass filter, whose transmission coefficient in the Ka-band depends on the applied pressure, and two cross-polarized Rx- and Tx-antennas connected to the terminals of the filter for enhancing the full-scale measurement range of the sensor's Radar echo level. The filter is 3D-printed via the Sintering Laser Melting technique. For a Radar-to-sensor distance of 3 meters, a high full-scale measurement range of $8 \mathrm{~dB}$ on the echo level is reported with a measurement sensitivity of $0,03 \mathrm{~dB} / \mathrm{mbar}$ for applied pressure ranging from 350 mbar to 640 mbar.
\end{abstract}

Keywords - Passive sensors, wireless sensors, additive technology, microfluidic technology, field depolarization, remote sensing.

\section{INTRODUCTION}

Wireless and passive (or zero-power) sensors are commonly used for the short-range remote reading of physical quantities (such as, the temperature, humidity and pressure). The remote sensing can be performed by using well-known Radio Frequency Identification (RFID) or Surface Acoustic Waves (SAW) sensor tags. Because they are chipless and batteryless, these sensor tags are suitable for applications involving, e.g., extreme temperatures and/or very high pressures. However, the short reading range of passive wireless sensors (typically lower than 3 meters [1-3]) can be a strong limitation to their systematic application in harsh environments. For example, the reader might be degraded in extrem conditions such as by temperature or radioactivity.

To achieve both the reading and identification of passive sensors for large reader-to-sensor distances, the use of FrequencyModulated Continuous-Wave (FMCW) Radars was proposed very recently in [4]. Up to now, this Radar-based technique requires the fabrication of millimeter-wave sensing devices from costly clean room technological facilities (see, e.g., [5]). In order to reduce the fabrication cost, we investigate here the feasibility of designing such devices from using a 3D printing additive technology. The proof of concept is illustrated in this paper from the design, fabrication and Radar interrogation of a Ka-band microfluidic pressure sensor. The sensor is composed of a microfluidic band-pass filter, whose transmission coefficient in the Ka-band depends on the applied pressure. The filter is fabricated by using the Sintering Laser Melting technique.

The paper is organized in two sections. The first section described the microfluidic 3D-printed pressure sensor. In the second section, the sensor is remotely interrogated from a $24 \mathrm{GHz}$ FMCW Radar for various applied pressures.

\section{Microfluidic 3D Printed KA-BAND Pressure SENSOR}

The pressure sensor is designed to operate in the modulation bandwidth [22.8-24.8 GHz] of a FMCW Radar. It is composed of a band-pass rectangular waveguide (WR28 - 3.556 $\mathrm{mm}$ x $7.112 \mathrm{~mm}$ ) filter connected to a vertically (V) polarized antenna at one port $(\mathrm{Rx}$, port 1$)$ and to a horizontally $(\mathrm{H})$ polarized antenna at the second port (Tx, port 2). A polytetrafluoroethylene (PTFE) circular channel (relative permittivity of 2.1 , with inner and outer diameters of $300 \mu \mathrm{m}$ and $800 \mu \mathrm{m}$, respectively) crosses the waveguide in the region where the electric field magnitude of the fundamental $\mathrm{TE}_{10}$ mode is maximum (see Figure 1(a)).

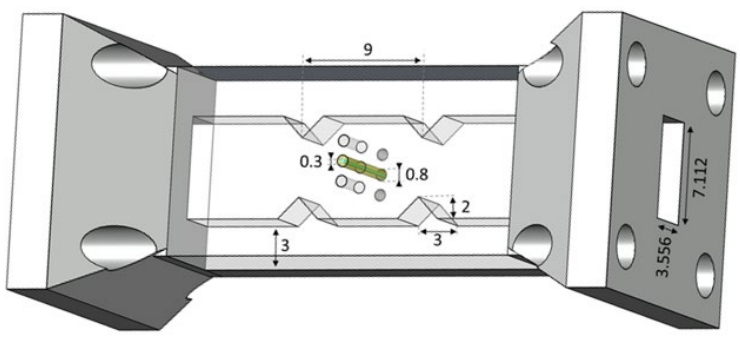

(a) 


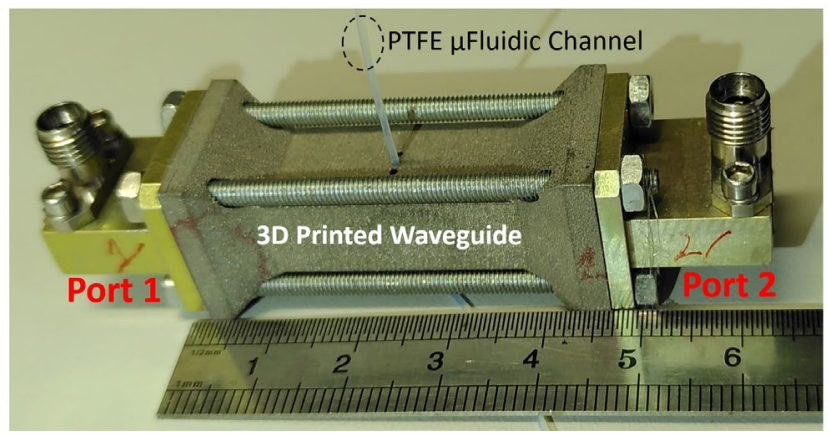

(b)

Fig. 1. Microfluidic pressure sensor (without its cross-polarized Rx- and Tx-antennas): (a) 3D view of the microfluidic tunable band-pass filter used as a pressure sensing device (dimensions are in millimeter) and, (b) Photo of the 3D-printed microfluidic filter fabricated via additive technology. Two SpaceK T28-K WR28to-coax adapters are used here to connect the V-polarized Tx-antenna at port 1 and H-polarized Rx-antennas at port 2.

A liquid (here we use water, with relative dielectric permittivity of 38 and loss tangent of 1 [5]) flows inside the channel. As the pressure increases, the liquid level moves inside the channel and gradually modifies the transmission coefficient $\mathrm{S}_{21}$ of the bandpass filter. As a result, the electromagnetic power received by the V-polarized Rx-antenna of the sensor is more or less attenuated, depending on the liquid level in the channel, which in turn is a function of the applied pressure at one end of the channel. The electromagnetic power is then re-radiated by the H-polarized Tx-antenna of the sensor, and part of this power is received by the Rx-antenna of the Radar. Following [2], the electric fields received and re-radiated by the sensor are cross-polarized for mitigating the impact of the electromagnetic clutter on the measurement accuracy of sensor's Radar echo level. For this specific reason, the sensor is called here a depolarizing sensor.

The filter is fabricated via the Sintering Laser Melting technique (manufacturing precision of $40 \mu \mathrm{m}$ and surface roughness of $20 \mu \mathrm{m})$. The metal is stainless steel (316L, conductivity of $310^{6} \mathrm{~S} / \mathrm{m}$ ). As it can be observed from Figure 1(a), the filter is composed of a (unique) resonant cell with triangular metallic diaphragms in order to be compliant with the additive technological process. Figure 1(b) shows the fabricated microfluidic tunable band-pass filter used as a pressure sensing device (or transducer). The liquid displacement in the PTFE channel is controlled by adjusting the pressure at one end of the channel (with the Fluigent EZ-MFCS pressure controller), while the other end is closed. The applied pressure ranges from 0 bar to 1 bar (by step of 10 mbar) and the corresponding fluid level ranges from 0 to $15 \mathrm{~mm}$ inside the channel.

Let $\mathrm{T}_{\text {mean }}$ be the mean-value of the transmission coefficient $\left|\mathrm{S}_{21}\right|$ in the modulation bandwidth $[22,8 \mathrm{GHz}-24,8 \mathrm{GHz}]$ of the FMCW Radar, which will be used in Section III for the remote interrogation of the pressure sensor. Figure 2 reports the measured values of $\mathrm{T}_{\text {mean }}$ as a function of the liquid level inside the channel. The measurement sensitivity is found to be $2.3 \mathrm{~dB} / \mathrm{mm}$ and the

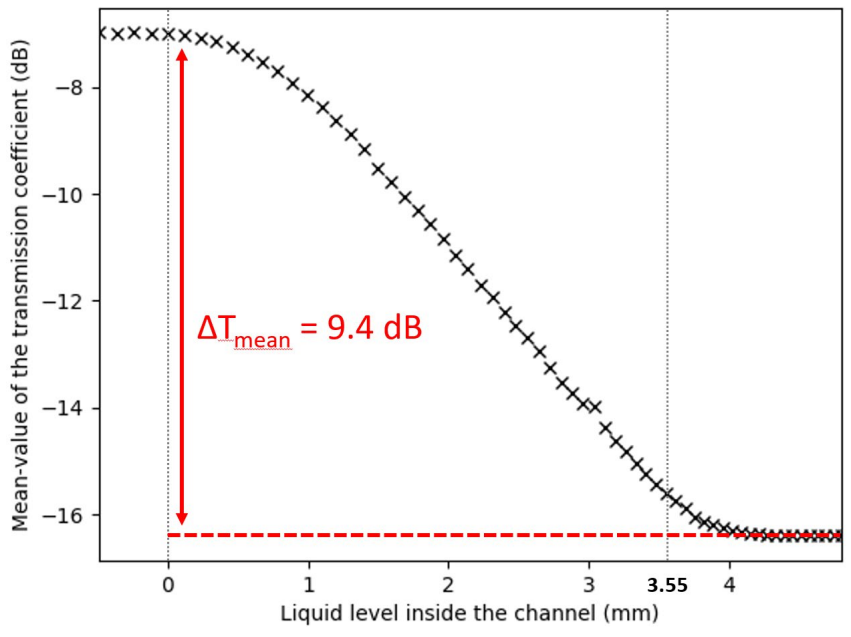

full-scale measurement range $\Delta \mathrm{T}_{\text {mean }}$ is of $9.4 \mathrm{~dB}$.

Fig. 2. Mean-value $T_{\text {mean }}$ of the measured transmission coefficient $\left|\mathrm{S}_{21}\right|$ of the microfluidic filter in the FMCW Radar modulation bandwidth [22.8 GHz - $\left.24.8 \mathrm{GHz}\right]$ as a function of the liquid (water) level inside the channel. As the pressure increases, the liquid progressively fills the PTFE channel crossing the waveguide section (in the region where the magnitude of the $\mathrm{TE}_{10}$-mode electric field is maximum) and gradually modifies the transmission coefficient of the filter.

\section{Wireless MEASUREMENT OF THE PRESSURE FROM THE RADAR ECHO OF THE 3D-PRINTED PASSIVE SENSOR}

The indoor experimental setup for the remote measurement of the pressure is shown in Figure 3. It is located in a room of our Laboratory. The wireless interrogation is performed by using a 24GHz FMCW Radar (model DK-sR-1030e from IMST GmbH [6]) 
which transmits a frequency modulated signal (or chirp) at a carrier frequency of $23.8 \mathrm{GHz}$. The modulation bandwidth is [22.8 $\mathrm{GHz}-24.8 \mathrm{GHz}]$ and consequently, the theoretical range resolution is of $7.5 \mathrm{~cm}$.

The front-end of the Radar uses one Tx-antenna and two Rx-antennas. The Tx-antenna is a V-polarized lens antenna (gain of $30 \mathrm{dBi}$ ) and following [4], two horn antennas (gain of $20 \mathrm{dBi}$ ) are used to receive the frequency-modulated (FM) fields re-radiated and backscattered by the 3D-printed sensor:

(i) one $\mathrm{Rx}$-antenna is H-polarized for deriving the pressure from the electric field re-radiated by the sensor;

(ii) the second Rx-antenna is V-polarized for locating the sensor from the electric field backscattered in this polarization by the sensor.

Moreover, in order to separate the time-of-arrival of the re-radiated and backscattered FM fields, delay lines (electrical length of $1.5 \mathrm{~m}$ ) are placed between the two ports of the filter and the sensor's Rx- and Tx-antennas, as shown in Figure 3(b). The distance between the Radar and the sensor is set here to 3 meters.

For each applied pressure, the Radar transmits a V-polarized FM electric field, which is received by the V-polarized Rx-antenna of the sensor, and is then re-radiated by the H-polarized Tx-antenna of this sensor. Before the re-radiation, the received FM field propagates inside the filter and is subjected to an attenuation, which depends on the pressure-dependent fluid level in the channel. The re-radiated FM field, whose magnitude is dependent on the applied pressure, is finally received by the Rx-antennas of the Radar.

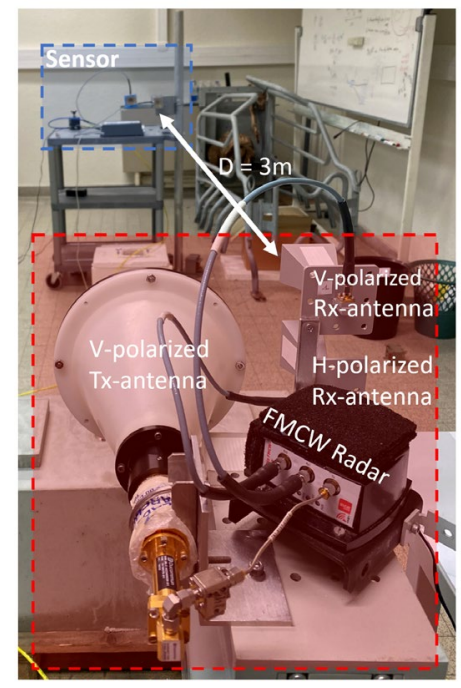

(a)

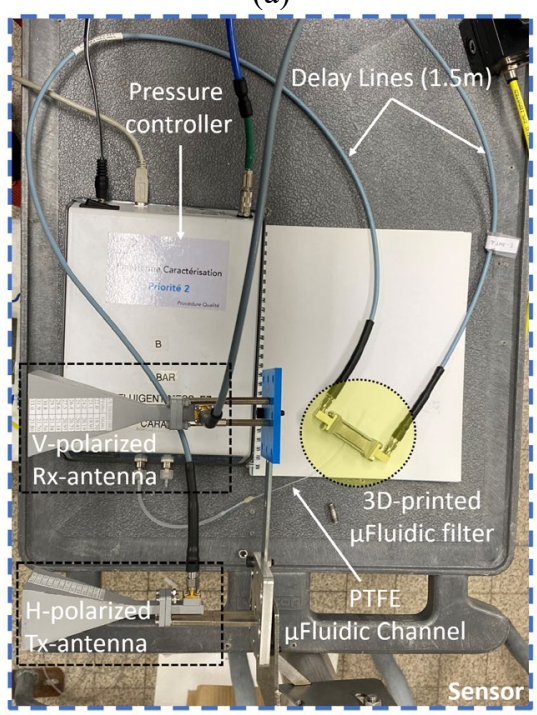

(b)

Fig. 3. (a) Photo of the indoor experimental setup and (b) Photo of pressure sensor combining the 3D-printed microfluidic filter, the two cross-polarized Tx- and Rx-antennas and the delay lines. 
In Figure 4 are displayed the mean value (over 100 measurements) and standard deviation of the echo level received by the Hpolarized Rx-antenna of the Radar. For an applied pressure at one end of the microfluidic channel ranging from 350 mbar to 640 mbar, the liquid level varies inside the waveguide. Outside this range (adjustable by design), the channel is empty or is completely filled with liquid. A convenient linear variation of the echo level (in $\mathrm{dB}$ ) is obtained as the applied pressure varies.

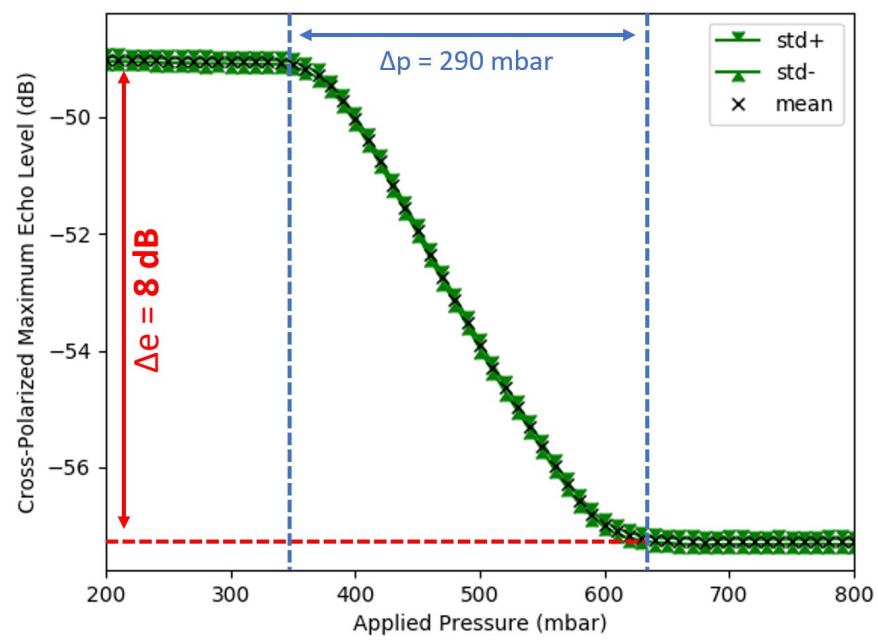

Fig. 4. Measured cross-polarized (V-to-H) Radar echo level of the 3D-printed microfluidic sensor as a function of the applied pressure. For pressure ranging from $350 \mathrm{mbar}$ to $640 \mathrm{mbar}$ the liquid level varies inside the waveguide. For a fixed applied pressure, the mean value and standard deviation of the Radar echo is computed from 100 successive echo measurements.

For a Radar-to-sensor distance of 3 meters, a high full-scale measurement range $\Delta \mathrm{e}$ of $8 \mathrm{~dB}$ on the echo level is obtained with a measurement sensitivity of $0.03 \mathrm{~dB} / \mathrm{mbar}$ for applied pressure ranging from $350 \mathrm{mbar}$ to $640 \mathrm{mbar}$. The precision of the measurement is derived from the calculated standard deviation. We obtain a maximal standard deviation of $0.07 \mathrm{~dB}$ for an applied pressure of 550 mbar. Hence, the standard deviation represents the precision of the measurement according to the signal-to-noise ratio of the radar. Knowing the sensitivity of the pressure sensor, the precision of the measurement is 3 mbar. This precision obtained at a Radar-tosensor distance of $3 \mathrm{~m}$ may be degraded at a higher range of interrogation, or if a mechanical beamscanning is performed [4]. The sensitivity of the proposed sensor can be compared with the one obtained in [4]. With identical reader and antennas and a reading distance of 4,2m, the current sensor had a sensitivity ten times higher and a dynamic range more than two times higher. Moreover, we have observed (not shown in this paper) that reading range up to $22 \mathrm{~m}$ is reachable by using the fabricated 3D-printed microfluidic sensor, but at the expense of lower measurement sensitivity. Anyway, these results are very encouraging and prove that additive technologies (such as the Sintering Laser Melting technique) can be advantageously used to manufacture wireless chipless sensors in Ka-Band.

\section{CONCLUSION}

In this paper, we investigated the feasibility of designing wireless and chipless sensors in the Ka band from using a 3D printing additive technology. We demonstrate that a 3D-printed microfluidic sensor allows the remote measurement of the pressure. A pressure measurement sensitivity of $0,03 \mathrm{~dB} / \mathrm{mbar}$ is obtained for a pressure variation of $290 \mathrm{mbars}$ and sensor-to-reader distance of 3 meters.

Additive technology (Sintering Laser Melting technique) in addition with microfluidics brings a promising solution for manufacturing cost-effective wireless passive sensors in the Ka-band. The next step consists of manufacturing millimeter-wave 3D sensors (combining microfluidic filters and antennas) from this technology and demonstrates the feasibility of their long-range wireless reading in various harsh environments.

\section{REFERENCES}

[1] M. A. Islam, N. Karmakar and A. K. M. Azad, "Aperture coupled UWB microstrip patch antenna array for mm-Wave chipless RFID tag reader," 2012 IEEE International Conference on RFID-Technologies and Applications (RFID-TA), Nice, 2012, pp. 208-211.

[2] P. Schumacher, C. Schuster, A. Jiménez-Sáez, M. Schüßler and R. Jakoby, "Passive chipless wireless pressure sensor for Harsh and reflective environments," 2018 11th German Microwave Conference (GeMiC), Freiburg, Germany, 2018, pp. 227-230

[3] A. M. J. Marindra and G. Y. Tian, "Chipless RFID Sensor Tag for Metal Crack Detection and Characterization," in IEEE Transactions on Microwave Theory and Techniques, vol. 66, no. 5, pp. 2452-2462

[4] D. Henry, T. Marchal, J. Philippe, P. Pons and H. Aubert, "Classification of Radar Echoes for Identification and Remote Reading of Chipless Millimeter-Wave Sensors," IEEE Transactions on Microwave Theory and Techniques, Oct. 2020.

[5] J. Philippe, M.V. De Paolis, D. Henry, A. Rumeau, A. Coustou, P. Pons, H. Aubert, "In-Situ Wireless Pressure Measurement Using Zero-Power Packaged Microwave Sensors," Sensors, Vol. 19, Issue 6, pp.1263-1274, March 2019. 
[6] U. Kaatze, V. Uhlendorf, "The Dielectric Properties of Water at Microwave Frequencies," Zeitschrift für Physikalische Chemie, Vol. 126. Issue 2, pp. 151$165,1981$.

[7] Products-IMST Radar. Accessed: Mar. 16, 2018. [Online]. Available: http://www.Radarsensor.com/products/ 